\title{
Towards Designer Microparticles: Simultaneous Control of Anisotropy, Shape, and Size
}

Srijanani Bhaskar, Kelly Marie Pollock, Mutsumi Yoshida, and Joerg Lahann*

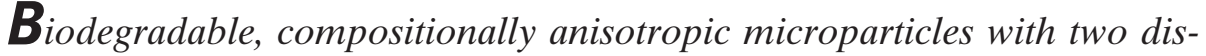
tinct compartments that exhibit controlled shapes and sizes are fabricated. These multifunctional particles are prepared by electrohydrodynamic cojetting of poly(lactide-co-glycolide) polymer solutions. By varying different solution and process parameters, namely, concentration and flow rate, a variety of non-equilibrium bicompartmental shapes, such as discoid and rod-shaped microparticles are produced in high yields. Optimization of jetting parameters, combined with filtration, results in near-perfect, bicompartmental spherical particles in the size range of 3-5 $\mu \mathrm{m}$. Simultaneous control over anisotropy, size, shape, and surface structure provides an opportunity to create truly multifunctional microparticles for a variety of biological applications, such as drug delivery, diagnostic assays, and theranostics.
\end{abstract}

\section{Introduction}

Custom-tailored nano- and microparticles with potential use for biomedical applications, such as targeted drug delivery or medical imaging, require narrowly controlled chemical compositions as well as precisely engineered physical properties. ${ }^{[1]}$ Hence, materials scientists have increasingly sought to

[*] Prof. J. Lahann

Departments of Chemical Engineering, Materials Science and Engineering

and Macromolecular Science and Engineering

University of Michigan

Ann Arbor, MI 48109 (USA)

E-mail: lahann@umich.edu

S. Bhaskar

Macromolecular Science and Engineering Program

University of Michigan

Ann Arbor, MI 48109 (USA)

K. M. Pollock

Department of Chemical Engineering

Cornell University

Ithaca, NY 14853 (USA)

Dr. M. Yoshida

Department of Chemical Engineering

University of Michigan

Ann Arbor, MI 48109 (USA)

DOI: $10.1002 / \mathrm{smll} .200901306$ delineate materials effects by considering physical particle attributes, such as size, surface microstructure, roughness, or anisotropy of particles without altering the particle chemistry. ${ }^{[1]}$ For instance, control over particle shape has been demonstrated previously via photolithographic techniques, ${ }^{[2]}$ fabrication in non-wetting templates, ${ }^{[3]}$ and modification of spherical particles. ${ }^{[4]}$ On the other hand, examples of size control include microfluidic ${ }^{[5]}$ and emulsion polymerization ${ }^{[6]}$ methods. Although colloidal structures showing individual control over shape, ${ }^{[4]}$ size, anisotropy,${ }^{[7]}$ and surface chemistry have been previously fabricated, traditional fabrication processes are best suited to control one of these parameters at a time. The establishment of versatile particle fabrication processes that enable simultaneous control of several physical attributes remains a key challenge in the biomedical arena. ${ }^{[8]}$ We now report on the fabrication of multifunctional particles based on electrohydrodynamic co-jetting, whereby appropriate selection of process parameters allows for variation of the three basic physical attributes: anisotropy, shape, and size.

We previously demonstrated control over particle anisotropy at the nanometer scale by fabricating bi- and tricompartmental micro- and nanostructures via electrohydrodynamic co-jetting of two (or three) aqueous polymeric solutions. ${ }^{[7,9-11]}$ More recently, we extended this process to biodegradable particles and fibers with multiple independent compartments, which were prepared by co-jetting of organic polymer solutions. ${ }^{[12-14]}$ Typically, two or more jetting solutions are pumped through a side-by-side capillary system 
under laminar flow. Similar to conventional electrospinning, the application of an electric potential results in distortion of the pendant droplet into a Taylor cone. ${ }^{[15]}$ Stretching of the jet results in formation of well-defined particles through rapid solvent evaporation. ${ }^{[16,17]}$ A fine interplay of forces, such as attractive electrical forces and surface tension, combined with

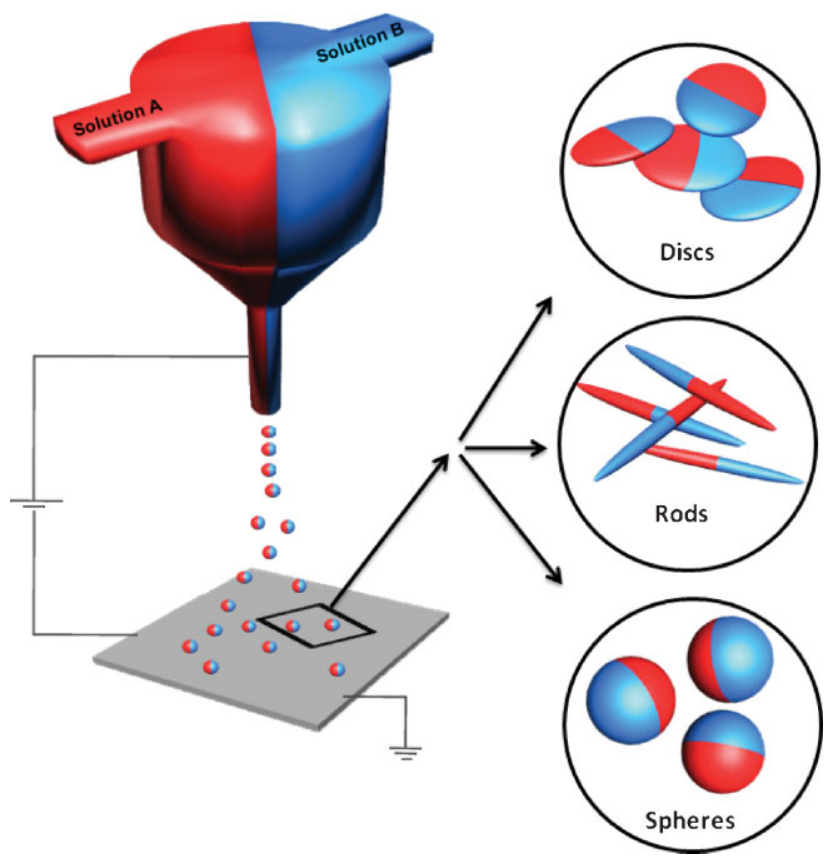

Figure 1. Schematic image of the electrohydrodynamic co-jetting process yielding bicompartmental spherical, discoid, and rod-shaped microparticles. fast solvent evaporation is necessary to enable formation of microparticles via electrohydrodynamic co-jetting. While electrohydrodynamic co-jetting typically results in fibers or spherical particles, precise orchestration of process parameters may offer the distinct possibility to obtain a wider range of particle shapes. This is schematically depicted in Figure 1. Several examples of control over particle shape and surface morphology via manipulation of different jetting solution and process parameters exist, notably the production of tapered, porous, and blood-cell-shaped particles made from poly(lactide-co-glycolide) (PLGA), ${ }^{[16]}$ controlled fabrication of biodegradable collapsed and spherical microstructures, ${ }^{[18,19]}$ cup-shaped polystyrene and poly(methyl methacrylate) particles, ${ }^{[20]}$ and also drug-loaded particles of controllable shapes. ${ }^{[21]}$

\section{Results and Discussion}

Here, we systematically investigate the effect of process parameters on particle shape and morphology during electrohydrodynamic co-jetting of biodegradable PLGA polymers from organic solvents. Initially, we focused on the concentration of the PLGA-containing jetting solutions. Starting at the lower concentration boundary, defined by the ability to support a stable jetting cone, electrohydrodynamic co-jetting of a $1.3 \%$ w/w solution (in 95:5 v/v chloroform: dimethylformamide (DMF)) of each polymer (PLGA 85:15 and PLGA 50:50), at a flow rate of $0.15 \mathrm{~mL} \mathrm{~h}^{-1}$, yielded discoid biphasic particles (Figure 2a). Confocal laser scanning microscopy (CLSM) revealed that the bicompartmental nature of the particles was extremely well preserved, as indicated by the near-perfect "half-half" anisotropy of the disks shown in Figure 2a. The

(a)

(b)

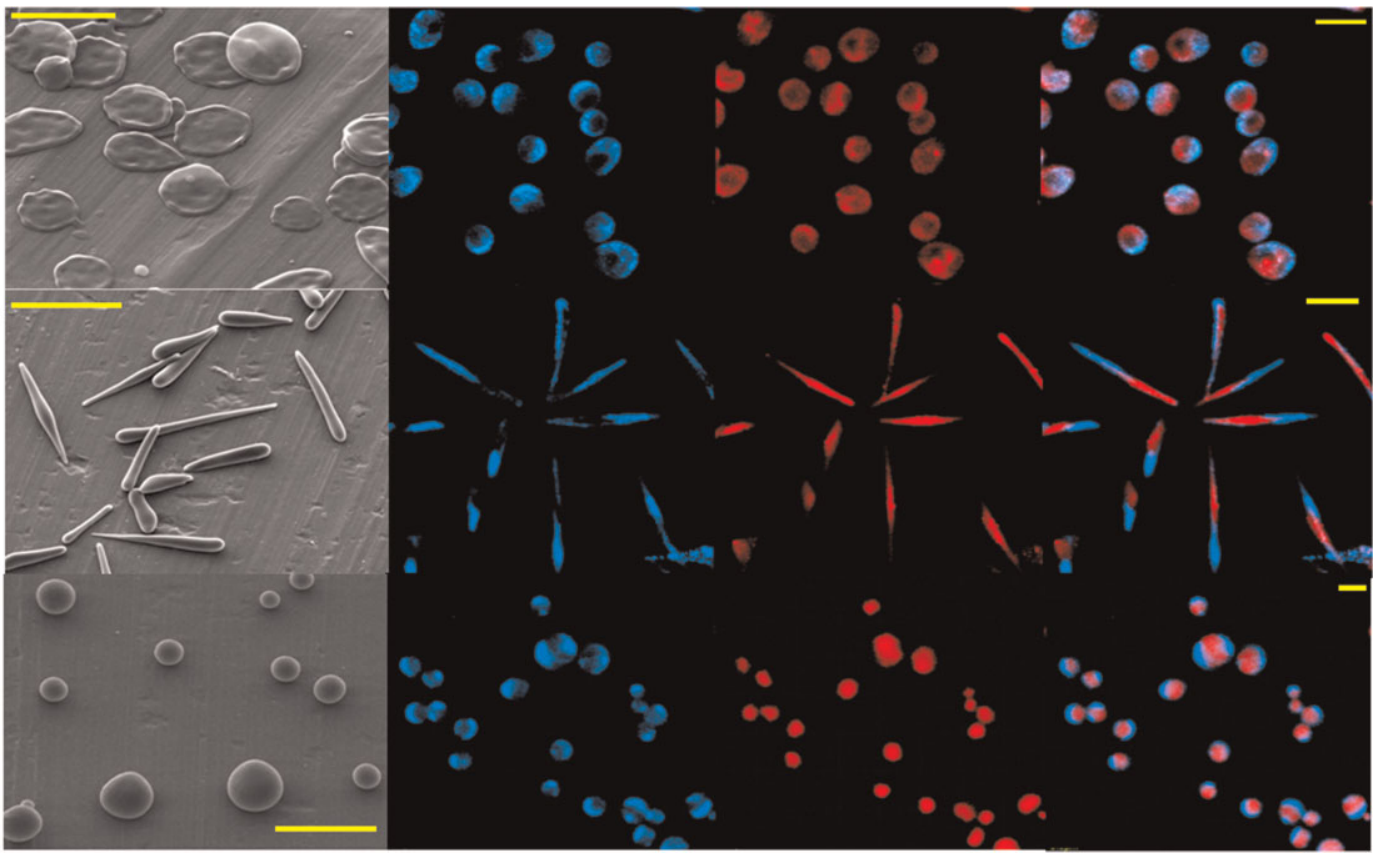

Figure 2. SEM and CLSM images of bicompartmental particles of different shapes, a) discs, b) rods, and c) spheres made from PLGA polymers via electrohydrodynamic co-jetting. Blue fluorescence represents ADS406PT and red fluorescence represents ADS306PT. Individual blue and red CLSM images are shown, followed by their overlay. Scale bars represent a) $5 \mu \mathrm{m}$, b) $20 \mu \mathrm{m}$ for SEM and $10 \mu \mathrm{m}$ for CLSM, and c) $10 \mu \mathrm{m}$ for SEM and $5 \mu \mathrm{m}$ for CLSM. 
pronounced compartmentalization is reflective of an inherent stability of the Taylor cone, in spite of more complex evaporation processes encountered during co-jetting from chloroform and DMF mixtures. Interestingly, PLGA discs with a range of different surface morphologies were observed including very smooth and slightly rough discs. The latter displayed increased surface roughness predominantly as surface ridges as determined by scanning electron microscopy (SEM). Both the discoid shape and the uneven surface morphology of the bicompartmental microcolloids may be attributed to the rapid collapse of particles during solidification. Because the jetting solutions used for electrohydrodynamic jetting are dilute polymer solutions, particles will have to undergo substantial shrinkage during the transition from liquid droplets to solid particles. If particle shrinkage prior to solidification is gradual and homogenous throughout the particle, close-to-perfect spheres will be formed. If, however, rapid solvent evaporation occurs during jetting, the polymer concentration at the surface of the solidifying droplet will be higher than in the core. This difference in the evaporation rates arises from the difference in solution concentration, with solutions of lower concentration $(1.3 \% \mathrm{w} / \mathrm{w})$ showing a greater propensity to rapidly form a "skin" of polymer at the outer surface, compared to solutions with a higher concentration $(4.3 \%)$, wherein the skin formation is also accompanied by a gradual deposition of polymer in the core. Thus, for the jetting solutions with lower concentrations, higher polymer concentrations may be quickly established in the outside region of the droplet resulting in localized polymer precipitation. This preferential solidification in the shell region further inhibits solvent evaporation in the core and gives rise to an anisotropic particle formation mechanism. Ultimately, solvent will also evaporate from the core of the capsules creating a void in the particle center that causes the initially spherical particles to collapse. ${ }^{[18,22]}$ Consequentially, discoid particles, such as the microparticles shown in Figure 2a, can be formed. This hypothesis is supported by the fact that the peripheral portions of the discs are of relatively greater thickness and density in comparison with the inner areas, as visualized in SEM images (Figure 2a). Similar to the bicompartmental particles, monophasic jetting experiments performed with the same PLGAbased jetting solutions at comparable flow rates yielded discs as well (Supporting Information, Figure S1). In order to rule out the possibility that these structures might have resulted from the collapse of the droplet on the surface of the substrate owing to insufficient time for evaporation, a series of control experiments was carried out, where the distance between the capillary and the substrate was systematically varied. Independent of the distance between needle and counter electrode, all jetting experiments yielded discoid shapes, suggesting that the shape setting occurred during solvent evaporation in the jet and not during contacting the counter electrode (Supporting Information, Figure S2). Moreover, subtle variations of the solvent and flow rate yielded disc-shaped particles of different sizes and surface morphologies (Supporting Information, Figure S3).

In contrast, further increase of the PLGA concentration in both jetting solutions to $3.4 \%(\mathrm{w} / \mathrm{w})$, while maintaining flow rates of about $0.45 \mathrm{~mL} \mathrm{~h}^{-1}$, yielded predominantly rod-shaped biphasic particles (Figure 2b). Confocal micrographs reveal a mixture of different compartment architectures. A typical particle population included bicompartmental rods with compartments along their radial axis as well as particles with compartments oriented along the longitudinal axis (Figure 2b and Supporting Information, Figure S4). In addition, rodshaped microparticles with one compartment sandwiched in between the other were also observed (Supporting Information, Figure S4). In contrast to spheres and discs, microrods originate from droplets that are solidified before the droplets can assume their spherical form, that is, the solidification is so rapid that the filamentous region that connects successive droplets ${ }^{[23]}$ is partly retained during the jet breakup, giving rise to a rod-shaped configuration. This is also reflected in the SEM images of the rods (Figure 2b), some of which are tapered towards the end, and others that are spindle shaped, indicative of solidification via rapid solvent evaporation at various time points during jet defragmentation. In this respect, rodlike particles constitute a link between microspheres and microfibers.

In general, microrods were typically more heterogeneous than particles and disks and were typically accompanied by a minor fraction of spherical particles, possibly arising from secondary droplets due to higher flow rates. ${ }^{[23]}$ The addition of a small amount of triethylamine (3.6 vol. \% of solvent) as cosolvent yielded the most reliable formation of biphasic microrods in our hands. In addition to the use of triethylamine as co-solvent, the appropriate choice of flow rates was another essential factor for the formation of rods. If the same PLGA solutions were jetted at flow rates below $0.45 \mathrm{~mL} \mathrm{~h}^{-1}$, bicompartmental discs were predominantly obtained. Furthermore, lowering the PLGA concentrations from $3.4 \%(\mathrm{w} / \mathrm{w})$ to $1.3 \%(\mathrm{w} / \mathrm{w})$, while maintaining the same flow rates of $0.45 \mathrm{~mL}$ $\mathrm{h}^{-1}$ yielded biphasic discs, not rods (albeit of a larger diameter). Taken together, these data suggest the existence of an optimum concentration/flow rate/volatility combination, which favors formation of biphasic rod-shaped particles. As a matter of fact, the co-jetting experiments with two jetting solutions in a sideby-side configuration were repeated with a single jetting solution processed through a single nozzle under otherwise identical jetting conditions. This monophasic jetting did not result in the formation of majority of rods, implying that the formation of rods might be a unique aspect of electrohydrodynamic co-jetting using the unique side-by-side jetting configuration described herein.

Upon further increase of the PLGA concentrations to $4.3 \%(\mathrm{w} / \mathrm{w})$, close-to-perfectly spherical bicompartmental microparticles were obtained (Figure 2c). The larger polymer concentration favors isotropic solvent evaporation and gives raise to almost perfect spherical shapes. In general, increased PLGA concentrations lead to a higher solution viscosity, resulting in a decreased propensity of jet-breakup and increased droplet sizes. In this scenario, reduction of the surface-to-volume ratio causes more isotropic solvent evaporation, which drives the homogenous solidification that yields bicompartmental particles. In contrast, rapid evaporation rates typically associated with the higher surface-to-volume ratios of smaller droplets may result in anisotropic evaporation and, hence, can yield non-equilibrium shapes, such as discs. Furthermore, increased viscosities might result in increased chain-entanglement effects in the droplet, inhibiting polymer 
Table 1. Electrohydrodynamic processing and solution parameters yielding particles of different shapes.

\begin{tabular}{lccc}
\hline & Concentration $^{[\mathrm{a}]}$ & Flow rate $\left[\mathrm{mL} \mathrm{h}^{-1}\right]$ & ${\text { Triethylamine }[\%]^{[\mathrm{b}]}}$ \\
\hline Discs & 1.3 & 0.15 & - \\
Rods & 3.4 & 0.4 & - \\
Spheres & 4.3 & 0.17 & 3.6 \\
\hline
\end{tabular}

[a] Concentration in wt.\%. [b] Concentration in vol\% of solvent.

migration to the surface, thereby imparting higher structural stability to the core, further contributing to the formation of spherical shapes. ${ }^{[21]}$ Specific jetting conditions that lead to different shapes are summarized in Table 1 . With respect to compartmentalization within individual microspheres, it is noteworthy that "sandwich" type anisotropies were observed in addition to the previously observed half-half phase distribution. $^{[13]}$

In addition to shape, particle size is a critical base property for many biomedical applications. To better understand differences in the size and size distributions of particles made by electrohydrodynamic co-jetting, we conducted a detailed statistical analysis of spheres, disks, and rods, on the basis of SEM. Size-distribution data for diameters of different types of bicompartmental particles are shown in Figure 3. The histograms were obtained by analysis of SEM images of a larger population of particles directly after jetting. ${ }^{[24]}$ The discs varied from circular to slightly elongated, and exhibited an average diameter of $3.41 \pm 0.72 \mu \mathrm{m}$ (Figure $3 \mathrm{a}$ ). The latter corresponds to an average particle volume of $2.05 \pm 0.01 \mu \mathrm{m}^{3}$.
The volume was calculated based on a disk thickness of $0.225 \pm 0.03 \mu \mathrm{m}$, which was estimated based on SEM images. When prepared from jetting solutions with a higher polymer concentration, but with similar flow rates, microspheres showed an average diameter of $3.05 \pm 1.21 \mu \mathrm{m}$ (Figure $3 \mathrm{~d}$ ) and an average particle volume of $14.85 \pm 0.92 \mu \mathrm{m}^{3}$. The relatively broad size distributions may in this case be attributed to lack of long-term stability of the Taylor cone. In the case of rods, the average lengths of $18.37 \pm 6.17 \mu \mathrm{m}$ (Figure $3 \mathrm{~b}$ ) and average aspect ratios of $9.00 \pm 3.69 \mu \mathrm{m}$ (Figure $3 \mathrm{c}$ ) indicate preferential fabrication of larger particles. These rod dimensions translated into larger average particle volumes of $60.15 \pm 13.55 \mu \mathrm{m}^{3}$. The larger particle volumes may be attributed to the higher flow rates employed during electrohydrodynamic co-jetting of rods. Polydispersity of rodlike particles with respect to rod lengths and diameters is an indication of an increasingly randomized jet break-up mechanism.

In order to quantify the biphasic character of the microparticles, flow cytometry was performed for a representative population of bicompartmental particles (Figure 3). ${ }^{[10]}$ Prior to analysis of bicompartmental particles, a number of different reference groups were obtained including particles without dye, particles with ADS406PT in one compartment only, and particles with ADS306PT dye in one compartment only. During these experiments, the dye concentrations of all jetting solutions were maintained equal. After these reference samples were obtained and analyzed by flow cytometry, a fourth sample of particles, containing ADS306PT in one compartment and ADS406PT in the other compartment was investigated. Figure $4 \mathrm{a}-\mathrm{d}$ shows the four dot plots of the corresponding particles. Being negative for both the dyes, sample (i) fell in the lower left quadrant (Figure 4a). Microparticles loaded with one dye only, that is, sample groups (ii) and (iii), were characterized by fluorescence signals that were clearly confined to their respective quadrants (Figure 4b and c). Sample group (iv), however, was indicated by fluorescence signals located almost exclusively in the upper right quadrant (Figure 4d), confirming the presence of both dyes. Furthermore, sample (iv) showed excellent correlation between fluorescent intensities of ADS306PT and ADS406PT, which exhibit an almost linear relationship, indicating that the relative sizes of the compartments were retained irrespective of particle size. Based on combined qualitative and quantitative data from the CLSM micrographs and flow cytometry analysis, respectively, close to a $100 \%$ of the particle population appears to be bicompartmental (detailed information, including gating and threshold values and forward scatter and side scatter plots (FSC and SSC plots), is provided in the Supporting Information, Figure S5).

After the quantitative analysis of compartmentalization, we turned our intention to optimization of particle shape and size. We therefore investigated the formation of optimized spherical particles with two compartments in distribution. 

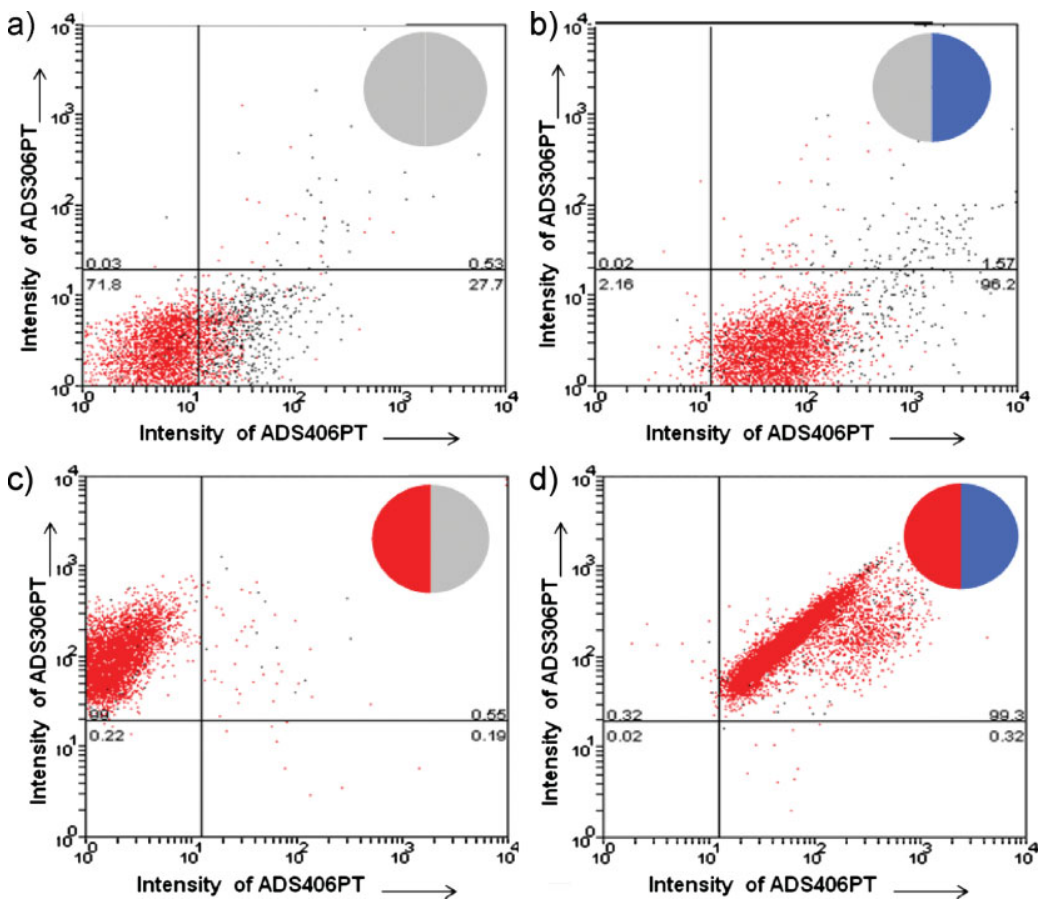

Figure 4. Flow cytometry anaylsis of spherical bicompartmental microparticles. Inset indicates the nature of dye loading in the particle. a) Bicompartmental particles fabricated without any dyes. These exhibit low ADS306PT and ADS406PT fluorescence intensities and fall in the lower left quadrant. b) Bicompartmental particles loaded with ADS406PT in one compartment and no dye in the other; these fall in the lower right quadrant. c) Particles loaded with ADS306PT in one compartment and no dye in the other, which appropriate exhibit high ADS306PT intensities and lie in the top left quadrant. d) Microparticles with ADS306PT in one compartment and ADS406PT in the other. Owing to high ADS306PT and ADS406PT intensities, these lie in the upper right quadrant.

further detail. For biological applications, such as drug delivery, control over particle size has been shown to impact their performance in vivo, by affecting several properties such as circulation times, clearance, penetration across biological barriers, and cellular uptake. ${ }^{[1]}$ In addition, particle aggregation and interaction with blood and tissue is also size dependent. Since similar types of particle may play a role in delivering therapeutics to antigen-presenting cells (APCs), which can uptake particles via phagocytosis, ${ }^{[25]}$ we chose to optimize bicompartmental microparticles to conform to a diameter range of $3-5 \mu \mathrm{m}$, a size range that has previously been shown to be effective in the delivery of genetic vaccines ${ }^{[26]}$ and immunosuppressants $^{[27]}$ to APCs, such as macrophages and dendritic cells. In order to achieve optimum uptake results, narrower size distributions are important, which could be obtained through development of effective filtering methods. With these goals in mind, we fine-tuned the co-jetting parameters and developed a suitable filtration protocol.

During electrohydrodynamic jetting, organic solutions are known to exhibit varicose instabilities to a lesser extent than their aqueous counterparts owing to their lower conductivities and dielectric con- a)

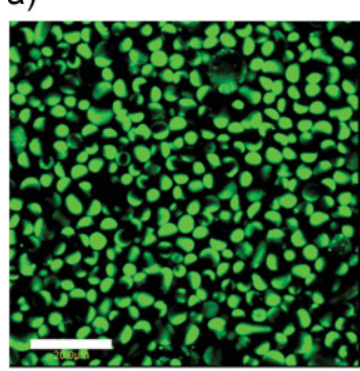
(c). All scale bars represent $20 \mu \mathrm{m}$. stants, thereby producing a lesser number of satellite droplets. This may potentially result in narrower and more uniform size distributions. ${ }^{[15]}$ On the other hand, the higher volatility of chloroform used in this study as solvent for electrohydrodynamic co-jetting gives rise to intermittently solidified jetting cones, which may result in larger particles. After establishing appropriate jetting solution conditions, which are of primary importance in determining shape and bicompartmental architecture, we evaluated process conditions, namely flow rate and voltage, for further optimization of size and shape. It is known that larger flow rates induce a greater number of satellite particles in the jet stream, ${ }^{[23]}$ resulting in reduced monodispersity. On the other hand, very low flow rates result in a droplet of higher viscosity due to a mismatch between rates of solvent evaporation and supply of fresh solution, giving rise not only to beaded fibers, fibers, and polydisperse particles in the population. For the desired diameter range, we found that a flow rate of $0.17 \pm 0.01 \mathrm{~mL} \mathrm{~h}^{-1}$ balanced the aforementioned issues. This was combined with a slightly higher operating voltage of $6 \pm 0.1 \mathrm{kV}$, which reduced the formation of donutlike shapes and ensured that the jet could be sustained for longer periods of time $(\approx 3 \mathrm{~h})$. Donutlike shapes, nonetheless, could not be removed completely, and microfiltration was subsequently employed for their removal. This approach resulted in milligram-scale particle quantities of close-to-perfect spheres, with excellent bicompartmental architecture, as seen in Figure 5. In particle populations obtained directly after jetting, donutlike shapes constituted approximately $8.2 \%$ of the entire population. These were completely eliminated via microfiltration, as seen in the low-magnification SEM images shown in Figure 6a. After filtration, $84 \%$ of the particle population was found to exhibit diameters in the range of 3 to $5 \mu \mathrm{m}$ (Figure 6c), compared to about $58 \%$ immediately after jetting, reflective of a uniformly sized particle population. The yield after filtration was found to be close to $60 \%$.

In electrohydrodynamic processing of polymer solutions, the physical attributes of particles, as well as the parameters b)

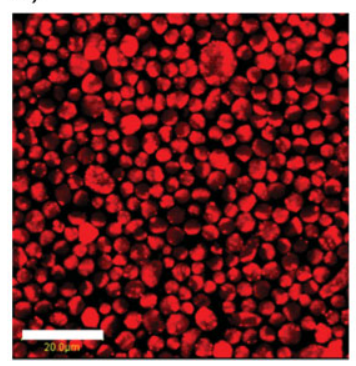

c)

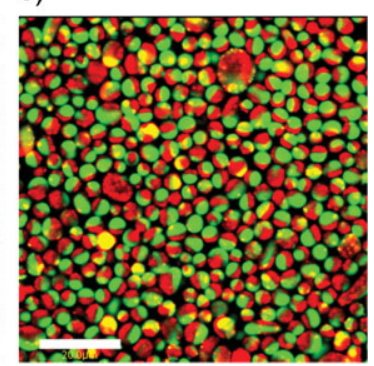

Figure 5. a-b) CLSM micrographs of bicompartmental microparticles after filtration. Green and red fluorescence depict PTDPV and ADS306PT, respectively, followed by the overlay 
(a)

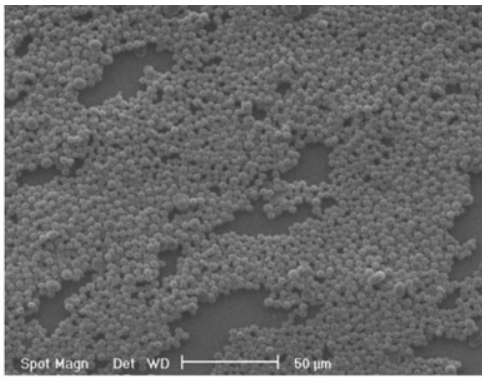

(b)

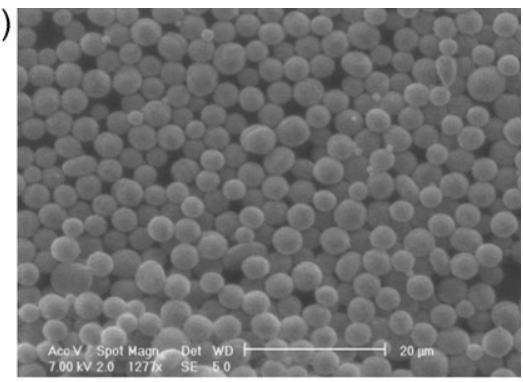

(c)

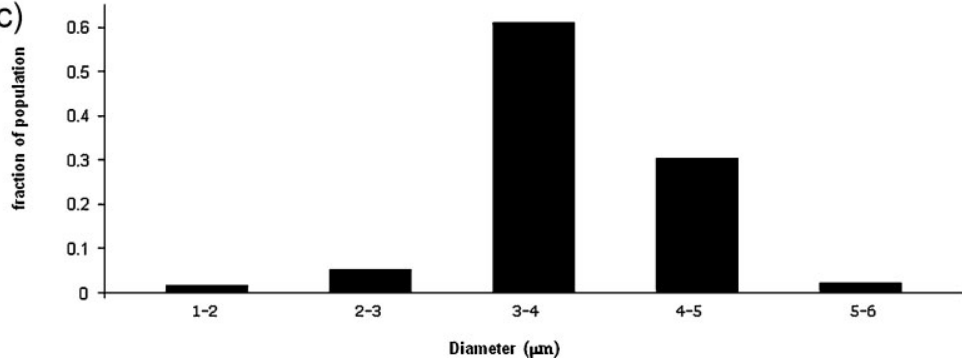

Figure 6. a,b) Low-and high-magnification SEM images of bicompartmental PLGA particles after filtration. A flow rate of $0.17 \mathrm{~mL} \mathrm{~h}^{-1}$ and a voltage of $6.1 \mathrm{kV}$ was employed in the co-jetting of a 4.3 wt. \% solution of PLGA 85:15 in 97:3 v/v chloroform: dimethylformamide. The donutlike flat shapes obtained during co-jetting were eliminated by filtration to yield optimized particles, with $84 \%$ of the total population in the size range of $3-5 \mu \mathrm{m}$, as shown in the size distribution in (c) obtained from representative SEM images.

that can be controlled to produce them, constitute a truly multidimensional design space. The controllable parameters can be based on the jetting solution, which includes surface tension, dielectric constant, electrical conductivity, density, and vapor pressure of the solvent, and the viscosity of the solution, which in turn depends on density and concentration of the polymer used. In addition, process parameters involve flow rate, current, separation distance between the electrodes, and diameter of the capillary. Furthermore, there are environmental variables that affect solvent evaporation, such as ambient temperature, pressure, and humidity. Simultaneous control over these variables may widen the access to particles with a multitude of sizes, shapes, surface morphologies and porosities. In electrohydrodynamic co-jetting of two or more solutions, the parameter space is complicated further due to the presence of a compound Taylor cone. ${ }^{[7]}$ Aiming to clearly delineate transitions with respect to shape and size, we further defined the effect of polymer concentration and flow rate on particle characteristics. This is depicted in Figure 7. At a given polymer concentration, an increase in solution flow rate results in increased particle size, due to an increase in radius of the jet, ${ }^{[16]}$ and hence increased droplet size in the jet stream. ${ }^{[23]}$ Thus, at a concentration of $1.3 \%$, increasing the flow rate from 0.15 to $0.7 \mathrm{~mL} \mathrm{~h}^{-1}$ resulted in an increase in disc diameter. Similarly, at a concentration of $4.2 \%$, flow rates of $0.17,0.35$, and $0.5 \mathrm{~mL} \mathrm{~h}^{-1}$, respectively, yielded bicompartmental microspheres that were correspondingly larger in diameter. On the other hand, polymer concentration directly influenced particle shape by affecting solution viscosity, which in turn influences jet break-up tendency and evaporation rate. Since solutions of higher viscosities resisted jet break-up, these conditions resulted in fibers. However, the effect was only observed at

low flow rates, when evaporation rates were much higher than solution flow rates. At these concentrations, however, as the flow rate is increased, a successive transition from fibers to beaded fibers, and then to distinct microspheres was observed. These observations may be attributed to an increase in volume of the primary droplet, which can enhance break-up even at higher viscosities. Thus, the same $7.4 \%$ PLGA solution resulted in fibers at a flow rate of $0.02 \mathrm{~mL} \mathrm{~h}^{-1}$ and beaded fibers at $0.04 \mathrm{~mL}$ $\mathrm{h}^{-1}$. However, a higher PLGA concentration of $10.7 \%$ resulted in distinct particles at flow rates as high as $0.7 \mathrm{~mL} \mathrm{~h}^{-1}$. As the concentration was further increased, the minimum flow rate required to produce distinct spheres also increased. Concentration was also found to influence the shape of discs because the discs seemed more "collapsed" when jetted from lower concentrations. This is most likely due to the presence of polymer at the core of the droplet at higher concentrations, which impedes microparticle collapse. Thus, for a given solvent system, individual or simultaneous control of flow rates and concentrations can be a straightforward yet extremely precise and effective means for obtaining particles of different sizes and shapes. The complexity that arises from co-jetting of two distinct solutions could also give rise to interesting effects, such as the rods observed herein. Choice of solvent is another aspect to controlling size and shape, as more volatile solvents can result in particles of larger diameter (Supporting Information, Figure S2).

\section{Conclusions}

In summary, we have demonstrated the fabrication of discoid, rodlike, and spherical bicompartmental biodegradable microparticles of different sizes via electrohydrodynamic cojetting. In addition, the effect of flow rate and concentration on shape and size was systematically studied. Solutions with lower concentrations (up to $3.4 \%$ ) yielded disc-shaped particles at flow rates from $0.02-0.7 \mathrm{~mL} \mathrm{~h}^{-1}$, whereas an increase in concentration $(4.5-12 \%)$ resulted in spherical particles. However, at higher concentrations, lower flow rates produced fibers and, as flow rate was further increased, beaded fibers and spheres. Rods were obtained at a concentration of $3.4 \%$ and at higher flow rates $\left(0.45 \mathrm{~mL} \mathrm{~h}^{-1}\right)$ upon addition of triethylamine. Flow rate was found to be a key variable affecting particle size, whereas concentration predominantly influenced particle shape, and also size (Figure 7). Spherical particles were further optimized with respect to size and shape via tuning of co-jetting parameters, followed by microfiltration to obtain monodisperse, spherical microparticles in the size range of $3-5 \mu \mathrm{m}$. Multifunctional microparticles with simultaneously controlled size, shape, and compartmentalization may have applications in drug delivery, cell targeting, and biomedical imaging. 


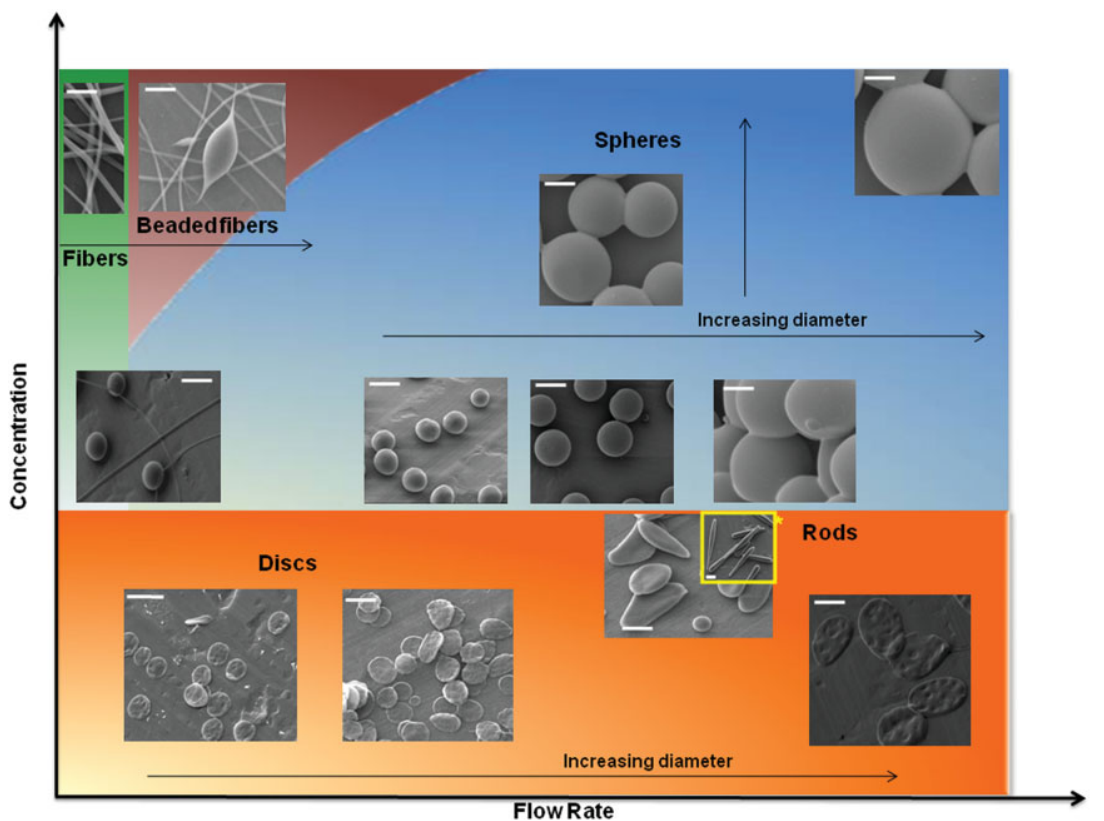

Figure 7. Schematic depiction of the effect of concentration and flow rate on size and shape of bicompartmental microparticles. At lower concentrations, bicompartmental discs are observed as a result of anisotropic solvent evaporation due to high surface-to-volume ratios afforded by small droplet sizes, which leads to the formation of a shell at the outer periphery, which collapses due to low overall polymer concentrations. This is observed even at higher flow rates, where particle shape is retained but the diameter of the discs increase due to larger droplet sizes, as shown by the orange gradient. As the concentration is increased, the shape is somewhat affected due to the availability of the polymerat the droplet core, which decreases the "flatness" of the discs. Rods, indicated by an asterisk, are observed at a critical concentration (3.4 $\mathrm{wt} \%$ ) and flow rate $\left(0.45 \mathrm{~mL} \mathrm{~h}^{-1}\right.$ ) but only upon the addition of triethylamine (3.6 vol\% of solvent). As concentration is increased, spheres are observed due to increased structural stability imparted by increased solution viscosities. Increasing the polymer concentration or flow rate leads to increased sphere diameters, depicted by the blue gradient. Fibers are observed at higher concentrations but at low flow rates. At higher concentrations and higher flow rates, a transition from fibers (green gradient), to beaded fibers (purple gradient), and then to particles is thus observed. All scale bars represent $5 \mu \mathrm{m}$.

\section{Experimental Section}

Materials: PLGA polymers with lactide:glycolide ratios of 85:15 $\left(M_{\mathrm{w}}\right.$ 40000-75000 $\left.\mathrm{g} \mathrm{mol}^{-1}\right)$ and 50:50 ( $M_{\mathrm{w}}$ 50000$75000 \mathrm{~g} \mathrm{~mol}^{-1}$ ), poly[tris(2,5-bis(hexyloxy)-1,4-phenylenevinylene)alt-(1,3-phenylenevinylene) (PTDPV), chloroform, N,N-dimethylformamide (DMF) up to $3.4 \%$ were purchased from Sigma-Aldrich, USA, and used as received. Polythiophene polymers, sold under commercial names ADS 306PT ( $M_{\mathrm{w}}$ 20000-70000 $\left.\mathrm{g} \mathrm{mol}^{-1}\right)$ ADS406PT $\left(M_{\mathrm{w}}\right.$ 30000-80000 $\left.\mathrm{g} \mathrm{mol}^{-1}\right)$ were purchased from American Dye Source, Canada.

Electrohydrodynamic jetting: A detailed description of the experimental setup for electrohydrodynamic co-jetting is provided elsewhere. ${ }^{[13]}$ Briefly, the two jetting solutions were pumped through a modified dual-capillary system (capillary diameter: 26 gauge, length: $8.2 \mathrm{~cm}$ ) held together in a side-by-side fashion. The capillaries were connected to the cathode of a DC voltage source (Gamma High Voltage Research, USA) and the flow rate was controlled via a syringe pump (Kd Scientific, USA). It should be noted that the flow rates reported correspond to the setting on the pump and due to flow from two capillaries the actual flow rate equals twice the value assigned to the pump. A flat piece of aluminum foil was used as a counter electrode, which also acted as a substrate for harvesting particles. The distance between the capillary tip and the substrate was maintained in the range of $28-33 \mathrm{~cm}$. All experiments were performed inside a fume hood at room temperature $\left(23^{\circ} \mathrm{C}\right)$. For filtration, particles were suspended in DI water containing $2 \% \mathrm{v} / \mathrm{v}$ Tween-20 to a final concentration of $1 \mathrm{mg} \mathrm{mL} \mathrm{m}^{-1}$. Filtration was performed using $40-\mu \mathrm{m}, 10-\mu \mathrm{m}$, and $5-\mu \mathrm{m}$ nylon mesh filters (Spectrum Labs, USA) in succession.

SEM and size-distribution analysis: Particles jetted on to the substrate were sputtered with gold and observed under a Philips XL30FEG ESEM scanning electron microscope (high-vacuum mode). In the case of filtered particles, $\approx 20 \mu \mathrm{L}$ of a concentrated aqueous suspension was cast on an SEM stub, and the water was allowed to evaporate at room temperature. Size-distribution analysis was performed on the SEM images using Image J software. ${ }^{[24]}$

CLSM: Glass coverslips were placed on top of the aluminum substrate during electrohydrodynamic co-jetting. The particles deposited on the coverslips were then examined using a confocal laser scanning microscope (Olympus Fluoview 500, Japan) at $100 \times$ magnification. ADS406PT, PTDPV, and ADS306PT were excited by 405-nm UV, 488-nm argon, and 533-nm helium-neon green lasers, respectively. Optical filters of emission wavelength $430-460 \mathrm{~nm}, 505-525 \mathrm{~nm}$, and 560-600 nm were used to visualize the fluorescence of ADS406PT, PTDPV, and ADS306PT, respectively.

Flow cytometry: $\mathrm{A} \approx 1 \mathrm{mg} \mathrm{mL}^{-1}$ particle suspension (loaded with the desired permutations of ADS306PT and ADS406PT dyes) was suspended in PBS and analyzed using a FACSDiVa Cell Sorter (BD Biosciences, 3-laser: 457/488/514 nm, $350 \mathrm{~nm}$, and $633 \mathrm{~nm}$ ). Signals from ADS406PT and ADS 306PT were resolved in two channels, i) excitation: 351-nm laser, emission: 424 $\pm 44-\mathrm{nm}$ bandpass filter), and ii) excitation: 488-nm laser, emission: $585 \pm 42-\mathrm{nm}$ bandpass filter. For each sample, 10000 events were collected. Data acquisition and analysis were performed using CellQuest Pro (BD Biosciences).

[1] S. Mitragotri, J. Lahann, Nat. Mater. 2009, 8, 15.

[2] D. Dendukuri, D. C. Pregibon, J. Collins, T. A. Hatton, P. S. Doyle, Nat. Mater. 2006, 5, 365.

[3] J. P. Rolland, B. W. Maynor, L. E. Euliss, A. E. Exner, G. M. Denison, J. M. DeSimone, J. Am. Chem. Soc. 2005, 127, 10096.

[4] J. A. Champion, Y. K. Katare, S. Mitragotri, Proc. Natl. Acad. Sci. USA 2007, 104, 1901. 
[5] S. Xu, Z. Nie, M. Seo, P. Lewis, E. Kumacheva, H. A. Stone, P. Garstecki, D. B. Weibel, I. Gitlin, G. M. Whitesides, Angew. Chem. Int. Ed. 2005 44, 724.

[6] K. P. Lok, C. K. Ober, Can. J. Chem. 1985, 63, 209.

[7] K. H. Roh, D. C. Martin, J. Lahann, Nat. Mater. 2005, 4, 759.

[8] S. C. Glotzer, M. J. Solomon, Nat. Mater. 2007, 6, 557.

[9] K. H. Roh, D. C. Martin, J. Lahann, J. Am. Chem. Soc. 2006, 128, 6796.

[10] K. H. Roh, M. Yoshida, J. Lahann, Langmuir 2007, 23, 5683.

[11] M. Yoshida, K. H. Roh, J. Lahann, Biomaterials 2007, 28, 2446.

[12] S. Bhaskar, J. Hitt, S. L. Chang, J. Lahann, Angew. Chem. Int. Ed. 2009, 48, 4589.

[13] S. Bhaskar, K. H. Roh, X. Jiang, G. L. Baker, J. Lahann, Macromol. Rapid Commun. 2008, 29, 1655.

[14] S. Bhaskar, J. Lahann, J. Am. Chem. Soc. 2009, 131, 6650.

[15] R. P. A. Hartman, D. J. Brunner, D. M. A. Camelot, J. C. M. Marijnissen, B. Scarlett, J. Aerosol Sci. 2000, 31, 65.

[16] C. Berkland, D. W. Pack, K. Kim, Biomaterials 2004, 25, 5649.

[17] Y. Q. Wu, R. L. Clark, J. Biomater. Sci. Polymer Ed. 2008, 19, 573.

[18] J. Xie, L. K. Lim, Y. Phua, J. Hua, C. H. Wang, J. Colloid Interface Sci. 2006, 302, 103.
[19] J. Yao, L. K. Lim, J. Xie, J. Huab, C. H. Wang, J. Aerosol Sci. 2008, 39, 987.

[20] J. Liu, S. Kumar, Polymer 2005, 46, 3211

[21] Y. Hong, Y. Lib, Y. Yin, D. Lia, G. Zou, J. Aerosol Sci. 2008, 39, 525.

[22] S. Koombhongse, W. Liu, D. H. Reneker, J. Polym. Sc. Part B: Polym. Phys. 2001, 39, 2598.

[23] R. P. A. Hartman, D. J. Brunner, D. M. A. Camelot, J. C. M. Marijnissen, B. Scarlett, J. Aerosol Sci. 2000, 31, 65.

[24] M. D. Abramoff, P. J. Magelhaes, S. J. Ram, Biophot. Int. 2004, 11, 36.

[25] D. S. Kohane, Biotechnol. Bioeng. 2007, 96, 203.

[26] S. R. Little, D. M. Lynn, Q. Ge, D. G. Anderson, S. V. Puram, J. Chen, H. N. Eisen, R. Langer, Proc. Natl. Acad. Sci. USA 2004, 101, 9534.

[27] S. Jhunjhunwala, G. Raimondi, A. W. Thomson, S. R. Little, J. Cont. Rel. 2009, 133, 191.
Received: July 22, 2009

Published online: November 20, 2009 